\title{
Research on Physical and Health Curriculum Evaluation Based on Ideal Grey Correlation Distance
}

\author{
Jiang Tao ${ }^{1}$, Zhou Xiao-feng ${ }^{2}$ and Cui Sheng-li ${ }^{3}$ \\ ${ }^{1,3}$ Sport Institute, Hebei Normal University, Shijiazhuang City, China \\ ${ }^{2}$ Tianjin Polytechnic University, Tianjin City, China \\ E-mail:fatima_pe@sina.com
}

\begin{abstract}
As the foundation and content, curriculum evaluation is an important part of education evaluation, having great significance in promoting reform and development of education, and improving the quality of education. With the continuous deepening of the reform of physical and health curriculum in our country, how to raise the scientific nature of physical and health curriculum evaluation become a bottleneck restricting the development of physical education. This study had a research on curriculum evaluation model based on grey ideal correlation distance of physical and health curriculum of 5 junior schools in Shijiazhuang with the literature material method, logic analysis and so on. The results show that $A_{1}$ belongs to type I, $A_{2}, A_{4}$ belong to type II, $A_{5}$ belongs to type III, $A_{3}$ belongs to type IV and in the type II, $A_{2}$ better than $A_{4}$. The 5 junior schools good and bad in sequence is No.2 middle school, No.9 middle school, No.28 middle school, Hebei Normal University affiliated middle school, and No15 middle school. The results of ideal grey relational distance curriculum evaluation model are consistent with actual situation and the further distinguish differences in the same type, effectively improve practicability and accuracy of physical and health curriculum evaluation.
\end{abstract}

Keywords: Physical and health curriculum; Evaluation; Grey correlation; Ideal solution; Euclidean distance

\section{Introduction}

Evaluation generally refers to specialized institutions or individuals that use the scientific methods, according to the evaluation criteria, analysis on objects and provide professional opinion, behaviors and process. Education evaluation is the content of school management, is national implementation of important methods for supervision and regulation. Curriculum evaluation is the key of education evaluation, it can diagnose scientifically curriculum construction present situation and exchange experience [1], to promote development and improve the overall quality of education is of great significance.

In 2005, our education fully implemented "physical and health curriculum standard", that is the beginning of physical and health curriculum reform [2]. However, some evaluations use more subjective language than quantitative description, lacking of scientific [3]. With crossing and integrating of different disciplines, the goal programming, analytic hierarchy process and multi-objective fuzzy decision [4-7] have greatly enriched the methods of physical and health curriculum evaluation. Ideal grey relational distance curriculum evaluation model, a new physical and health curriculum evaluation method is put forward on the basis of the gray system theories [8], ideal solution [9] and Euclidean distance [10]. It provides effective methods, to make scientific interpretations of "physical and health curriculum standard", to carry out the spirit of school physical and health curriculum evaluation, to strengthen the management and regulation of physical and health curriculum and to achieve self-improvement of physical 
and health curriculum. At the same time, to establish and improve physical and health curriculum evaluation mechanism are discussed in this paper.

\section{The Grey System Theory}

The grey system theory is founded by famous scholar DENG Ju-long in 1982, mainly for "limited sample", "poor information" uncertainty system as the research object, by means of the "part" of known information generation and development, to extract valuable information, to achieve a correct description of system behavior, evolution rule and effective monitoring [11]. The grey system theory mainly includes theory system, based on the grey dim sets, analysis system, based on grey relational space, methods system, based on grey sequence for GM as the core of the model system, with system analysis, evaluation, modeling, forecasting, strategic decision-making, control and optimization as the main body of technical system. At present, the grey system theory is widely used in industry, agriculture, economy, education, sports, and many other fields, It provides scientific methods for physical and health curriculum evaluation.

\section{The Ideal Grey Correlation Curriculum Evaluation Model}

According to distribution of confidence interval, whiten formula, fractional order approximation method and so on, to convert qualitative indexes into quantitative indexes of the physical and health curriculum evaluation system, the initial matrix of physical and health curriculum evaluation is:

$$
Y=\left(y_{i j}\right)_{m * n}=\left[\begin{array}{cccc}
y_{11} & y_{12} & \cdots & y_{1 n} \\
y_{21} & y_{22} & \cdots & y_{2 n} \\
\vdots & \vdots & \ddots & \vdots \\
y_{m 1} & y_{m 2} & \cdots & y_{m n}
\end{array}\right]
$$

\subsection{Idealization}

The idealization of initial matrix is to increase optimal and worst reference for physical and health curriculum evaluation scheme in initial evaluation matrix. The optimal physical and health evaluation scheme, the positive ideal evaluation scheme is the optimal value that each index of physical and health curriculum evaluation scheme can achieve; The worst physical and health evaluation scheme, the negative ideal evaluation scheme is the worst value that each index of physical and health curriculum evaluation scheme can achieve; Evaluation indexes are made of beneficial, damage, medium, in different ideal schemes, the methods of different types of indexes are also different.

\subsubsection{The Positive Ideal Initial Evaluation Matrix}

The $\mathrm{i}^{\text {th }}$ evaluation scheme in sequence of initial evaluation matrix

$$
y_{i}=\left(y_{i 1}, y_{i 2}, \ldots, y_{i n}\right), \quad \mathrm{i}=1,2 \ldots, \mathrm{m}
$$

To construct the optimal reference sequence

$\mathrm{L}_{\mathrm{j}}$ is beneficial index:

$$
y_{0}^{+}=\left(y_{01}, y_{02}, \cdots, y_{0 n}\right)
$$

$$
y_{0 j}=\max \left\{y_{1 j}, y_{2 j}, \cdots, y_{m j}\right\}
$$

$\mathrm{L}_{\mathrm{j}}$ is damage index:

$$
y_{0 j}=\min \left\{y_{1 j}, y_{2 j}, \cdots, y_{m j}\right\}
$$

$\mathrm{L}_{\mathrm{j}}$ is medium index:

$$
\mathrm{y}_{0 j}=y_{0 j}^{\prime}
$$


$y^{\prime}{ }_{0 j}$ is moderate attribute value of index $L_{j}$

Constructed matrix $\mathrm{Y}^{+}=\left(\mathrm{y}_{\mathrm{ij}}\right)(\mathrm{m}+1) \mathrm{n}$, there into, $\mathrm{i}=0,1,2, \ldots, \mathrm{m} ; \mathrm{j}=1,2, \ldots, \mathrm{n}$, as evaluation indexes of the positive idealized initial evaluation matrix.

\subsubsection{The Negative Ideal Initial Evaluation Matrix}

To construct the worst reference sequence

$\mathrm{L}_{\mathrm{j}}$ is beneficial index:

$$
\mathrm{y}_{0}^{-}=\left(y_{01}, y_{02}, \cdots, y_{0 n}\right)
$$

$\mathrm{L}_{\mathrm{j}}$ is damage index:

$$
y_{0 j}=\min \left\{y_{1 j}, y_{2 j}, \cdots, y_{m j}\right\}
$$

$\mathrm{L}_{\mathrm{j}}$ is medium index:

$$
y_{0 j}=\max \left\{y_{1 j}, y_{2 j}, \cdots, y_{m j}\right\}
$$

$$
y_{0 j}=\max \left\{\left|y_{1 j}-y_{0 j}^{\prime}\right|,\left|y_{2 j}-y_{0 j}^{\prime}\right|, \cdots,\left|y_{m j}-y_{0 j}^{\prime}\right|\right\}
$$

$\mathrm{y}^{\prime}{ }_{0 j}$ is moderate attribute value of index $\mathrm{L}_{\mathrm{j}}$

Constructed matrix $\mathrm{Y}^{-}=\left(\mathrm{y}_{\mathrm{ij}}\right)(\mathrm{m}+1) \mathrm{n}$, there into, $\mathrm{i}=0,1,2, \ldots, \mathrm{m} ; \mathrm{j}=1,2, \ldots, \mathrm{n}$, as evaluation indexes of negative idealized initial evaluation matrix.

\subsection{Standardization}

In ideal initial evaluation matrix, to eliminate incommensurability, it needs to apply dimensionless method to evaluating indexes, to get standardized evaluation matrix. For beneficial indexes of positive ideal initial evaluation matrix and damage indexes of negative ideal initial evaluation matrix standardizing by the following formula.

$$
p_{i j}=\frac{y_{i j}}{y_{0 j}}, i=0,1,2, \ldots, m ; j=1,2, \ldots, n
$$

$\mathrm{y}_{\mathrm{ij}}$ as initial sequence, $\mathrm{y}_{0 \mathrm{j}}$ as reference sequence

For damage indexes of positive ideal initial evaluation matrix and beneficial indexes of negative ideal initial evaluation matrix, making use of standardized function to conduct

$$
p_{i j}=\frac{y_{0 j}}{y_{\mathrm{i} j}}, i=0,1,2, \ldots, m ; j=1,2, \ldots, n
$$

$\mathrm{y}_{\mathrm{ij}}$ as initial sequence, $\mathrm{y}_{0 \mathrm{j}}$ as reference sequence

For medium indexes

$$
p_{i j}=\frac{\min \left(y_{i j}, y_{0 j}^{\prime}\right)}{\max \left(y_{i j}, y_{0 j}^{\prime}\right)}, i=0,1,2, \ldots, m ; j=1,2, \ldots, n
$$

$\mathrm{y}^{\prime}{ }_{0 \mathrm{j}}$ is the moderate attribute value of index

Ideal initial evaluation matrix $\mathrm{Y}^{ \pm}$gets the standardized ideal evaluation matrix $\mathrm{P}^{ \pm}$after standardized conducting.

\subsection{Incidence Matrix}

In ideal matrix of standardization, data column $\mathrm{y}_{0 \mathrm{j}}=\left(\mathrm{y}_{01}, \mathrm{y}_{02} \ldots, \mathrm{y}_{0 \mathrm{n}}\right)$ as the reference sequence; $\mathrm{y}_{1 \mathrm{j}}, \mathrm{y}_{2 \mathrm{j}}, \ldots, \mathrm{y}_{\mathrm{mj}}$ as the comparative sequence, $\beta_{\mathrm{ij}}=\left|\mathrm{y}_{0 \mathrm{j}}-\mathrm{y}_{\mathrm{ij}}\right|$, Define the $\mathrm{j}^{\mathrm{th}}$ index of the $\mathrm{i}^{\text {th }}$ evaluation scheme and the $\mathrm{j}^{\text {th }}$ index of reference sequence of correlation coefficient as

$$
\varepsilon_{i j}=\frac{\stackrel{\min \min }{\mathrm{j}} \beta_{i j}+\alpha \stackrel{\max \max }{\mathrm{i}} \beta_{i j}}{\beta_{i j}+\alpha \mathrm{m} \mathrm{i} \max \beta_{i j}}, i=0,1,2, \ldots, m ; j=1,2, \ldots, n
$$


Therefore, $\alpha$ is resolution ratio, $\alpha \in[0,1]$, and $\alpha=0.5$.

All row vector of standardized ideal evaluation matrix $\mathrm{P}^{ \pm}$relative to the optimal and the worst reference sequence respectively to get correlation coefficient, receive positive incidence matrix $\mathrm{E}^{+}$and negative correlation matrix $\mathrm{E}^{-}$.

$$
E^{+}=\left[\begin{array}{cccc}
\varepsilon_{01}^{+} & \varepsilon_{02}^{+} & \cdots & \varepsilon_{0 n}^{+} \\
\varepsilon_{11}^{+} & \varepsilon_{12}^{+} & \cdots & \varepsilon_{1 n}^{+} \\
\varepsilon_{21}^{+} & \varepsilon_{22}^{+} & \cdots & \varepsilon_{2 n}^{+} \\
\vdots & \vdots & \ddots & \vdots \\
\varepsilon_{m 1}^{+} & \varepsilon_{m 2}^{+} & \cdots & \varepsilon_{m n}^{+}
\end{array}\right], E^{-}=\left[\begin{array}{cccc}
\varepsilon_{01}^{-} & \varepsilon_{02}^{-} & \cdots & \varepsilon_{0 n}^{-} \\
\varepsilon_{11}^{-} & \varepsilon_{12}^{-} & \cdots & \varepsilon_{1 n}^{-} \\
\varepsilon_{21}^{-} & \varepsilon_{22}^{-} & \cdots & \varepsilon_{2 n}^{-} \\
\vdots & \vdots & \ddots & \vdots \\
\varepsilon_{m 1}^{-} & \varepsilon_{m 2}^{-} & \cdots & \varepsilon_{m n}^{-}
\end{array}\right]
$$

AHP, fuzzy clustering, expert testimony and other methods can be used in determining weight of index, sets of weight multiplied by each column of incidence matrix namely get a weighted incidence matrix.

\subsection{Degree of Correlation Distance}

In incidence matrix, the first line represents the optimal and the worst reference sequence. The article adopts the Euclidean distance as the standard measure. In positive and negative incidence matrix $\mathrm{E}^{ \pm}$, the value of Euclidean distance is calculated comparative sequence scheme relative to the optimal and the worst reference sequence.

$$
\mathrm{D}_{i}^{ \pm}=\sqrt{\sum_{j=1}^{n}\left(\varepsilon_{i j}^{ \pm}-\varepsilon_{0 j}^{ \pm}\right)^{2}}, i=1,2, \cdots, m
$$

According to the formula, we can get comparative sequence scheme relative to grey relational distance value of the optimal reference sequence $\mathrm{D}_{i}^{+}$and grey relational distance value of the worst reference sequence $\mathrm{D}_{\mathrm{i}}^{-}$. In practical curriculum evaluation, it often appears shown as in Figure 1.

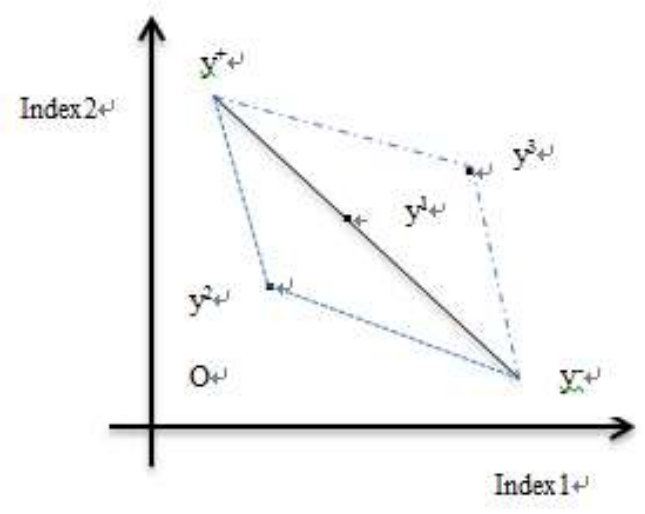

Figure 1. The Optimal and the Worst Reference Scheme

In Figure 1, y+ and y- respectively the optimal reference sequence scheme and the worst reference sequence scheme, $y^{1}$ is closer to the optimal reference scheme $y+$ than $y^{2}$ and $\mathrm{y}^{3}$, but at the same time, it is also closer to the worst reference scheme $\mathrm{y}^{-}$than $\mathrm{y}^{2}$ and $y^{3}$.In this case, we need to define a degree of grey correlation distance, its value represents virtue and defect degree of scheme. Define comparatives grey correlation distance $\mathrm{K}_{\mathrm{i}}$ to measure degree of comparative sequence scheme close to the optimal reference sequence and far from the worst reference sequence, comparative sequence scheme $y_{i}$ and the optimal reference sequence margin distance degree is $\mathrm{K}_{\mathrm{i}}$, the worst reference sequence margin distance degree is $\left(1-\mathrm{K}_{\mathrm{i}}\right)$. The grey correlation distance degree $\mathrm{K}_{\mathrm{i}}$ : 


$$
K_{i}=\frac{D_{i}^{+2}}{D_{i}^{-2}+D_{i}^{+2}}
$$

Ranking the value of grey correlation distance degree from small to large, the sort is on behalf of virtue and defect of comparative sequence ordering relation, the smaller the values of $K_{i}$, it shows that closer to the optimal scheme. The value of $K_{i}$ within $\{0,0.2\}$, evaluation type is I, within $\{0.2,0.5\}$, evaluation type is II, within $\{0.5,0.8\}$, evaluation type is III, within $\{0.8,1\}$, evaluation type is IV.

\section{Analysis on Physical and Health Curriculum Evaluation}

Physical and health curriculum evaluation as a complex gray system, the index is the fundamental basis and scale of evaluation. The index of curriculum evaluation system is basic specification, requirement and standard to carry out teaching jobs. Combines the condition of physical and health curriculum evaluation in Shijiazhuang city, to select teachers, field and equipment, schedules, exercise load, curriculum goals, curriculum implementation six indexes, make a detailed, system and comprehensive evaluation of physical and health curriculum. In these indicators, schedules are moderate indexes; the other indexes are positive indexes.

Based on physical and health curriculum evaluation of Shijiazhuang city, we extract physical and health curriculum of 5 junior middle school, (note: $A_{1}$ : NO. 2 junior middle school, $\mathrm{A}_{2}$ : NO. 9 junior middle school, $\mathrm{A}_{3}$ : NO. 15 junior middle school, $\mathrm{A}_{4}$ : NO. 28 junior middle school, $\mathrm{A}_{5}$ : the affiliated middle school of Hebei Normal University) the experts score directly to average, summarized in the table below:

Table 1. The Score of 5 Junior School Physical and Health Curriculum Evaluation

\begin{tabular}{ccccccc}
\hline name & teachers & field and equipment & load & schedules & goals & implementation \\
\hline $\mathrm{A}_{1}$ & 0.883 & 0.833 & 0.875 & 1.000 & 0.792 & 0.903 \\
$\mathrm{~A}_{2}$ & 0.805 & 0.805 & 0.836 & 0.941 & 0.813 & 0.896 \\
$\mathrm{~A}_{3}$ & 0.748 & 0.748 & 0.763 & 0.889 & 0.678 & 0.735 \\
$\mathrm{~A}_{4}$ & 0.739 & 0.739 & 0.840 & 0.889 & 0.785 & 0.872 \\
$\mathrm{~A}_{5}$ & 0.777 & 0.777 & 0.817 & 0.937 & 0.733 & 0.813 \\
\hline
\end{tabular}

Step1: According to expert evaluation scores, normalization, the initial matrix of physical and health curriculum evaluation

$$
\mathrm{Y}=\left|\begin{array}{llllll}
0.883 & 0.833 & 0.875 & 1.000 & 0.792 & 0.903 \\
0.805 & 0.805 & 0.836 & 0.941 & 0.813 & 0.896 \\
0.748 & 0.748 & 0.763 & 0.889 & 0.678 & 0.735 \\
0.739 & 0.739 & 0.840 & 0.889 & 0.785 & 0.872 \\
0.777 & 0.777 & 0.817 & 0.937 & 0.733 & 0.813
\end{array}\right|
$$

Step2: In above indexes, schedule is medium index, the other indexes are positive indexes. According to the formula (2)-(6), the positive ideal initial evaluation matrix is 


$$
\mathrm{Y}^{+}=\left|\begin{array}{llllll}
0.864 & 0.833 & 0.875 & 1.000 & 0.813 & 0.903 \\
0.864 & 0.833 & 0.875 & 1.000 & 0.792 & 0.903 \\
0.762 & 0.805 & 0.836 & 0.941 & 0.813 & 0.896 \\
0.719 & 0.748 & 0.763 & 0.889 & 0.678 & 0.735 \\
0.794 & 0.739 & 0.840 & 0.889 & 0.785 & 0.872 \\
0.723 & 0.777 & 0.817 & 0.937 & 0.733 & 0.813
\end{array}\right|
$$

Negative ideal initial evaluation matrix is

$$
\mathrm{Y}^{-}=\left|\begin{array}{llllll}
0.719 & 0.739 & 0.763 & 0.889 & 0.678 & 0.735 \\
0.864 & 0.833 & 0.875 & 1.000 & 0.792 & 0.903 \\
0.762 & 0.805 & 0.836 & 0.941 & 0.813 & 0.896 \\
0.719 & 0.748 & 0.763 & 0.889 & 0.678 & 0.735 \\
0.794 & 0.739 & 0.840 & 0.889 & 0.785 & 0.872 \\
0.723 & 0.777 & 0.817 & 0.937 & 0.733 & 0.813
\end{array}\right|
$$

Step3: Using different methods of standardization, we can get positive ideal evaluation matrix and negative ideal evaluation matrix

$$
\begin{aligned}
\mathrm{P}^{+} & =\left|\begin{array}{cccccc}
1 & 1 & 1 & 1 & 1 & 1 \\
1 & 1 & 1 & 1 & 0.947 & 1 \\
0.882 & 0.966 & 0.955 & 0.941 & 1 & 0.992 \\
0.832 & 0.898 & 0.872 & 0.889 & 0.834 & 0.814 \\
0.919 & 0.887 & 0.960 & 0.889 & 0.966 & 0.966 \\
0.837 & 0.933 & 0.934 & 0.937 & 0.902 & 0.901 \\
1 & 1 & 1 & 1 & 1 & 1 \\
0.832 & 0.887 & 0.887 & 0.889 & 0.856 & 0.814 \\
0.944 & 0.918 & 0.913 & 0.945 & 0.834 & 0.82 \\
1 & 0.988 & 1 & 1 & 1 & 1 \\
0.906 & 1 & 0.908 & 1 & 0.864 & 0.843 \\
0.994 & 0.951 & 0.934 & 0.949 & 0.925 & 0.904
\end{array}\right|
\end{aligned}
$$

Step4: In positive and negative ideal evaluation matrix, and different values of its optimal and worst curriculum scheme and all curriculum schemes remain to evaluate is

$$
\min _{i} \min _{j} \beta_{i j}=0, \max _{i} \max _{j} \beta_{i j}=0.186
$$

According to the formula (14), we can get the correlation coefficient of each curriculum scheme, forming positive ideal grey correlation curriculum evaluation matrix $\mathrm{E}^{+}$and negative ideal grey correlation curriculum evaluation matrix $\mathrm{E}^{-}$

$$
\begin{gathered}
\mathrm{E}^{+}=\left|\begin{array}{cccccc}
1 & 1 & 1 & 1 & 1 & 1 \\
1 & 1 & 1 & 1 & 0.781 & 1 \\
0.441 & 0.732 & 0.674 & 0.612 & 1 & 0.921 \\
0.356 & 0.477 & 0.421 & 0.456 & 0.759 & 0.333 \\
0.534 & 0.451 & 0.699 & 0.456 & 0.732 & 0.732 \\
0.363 & 0.581 & 0.585 & 0.569 & 0.487 & 0.484
\end{array}\right| \\
\mathrm{E}^{-}=\left|\begin{array}{cccccc}
1 & 1 & 1 & 1 & 1 & 1 \\
0.356 & 0.451 & 0.451 & 0.456 & 0.392 & 0.333 \\
0.624 & 0.531 & 0.517 & 0.628 & 0.359 & 0.341 \\
1 & 0.886 & 1 & 1 & 1 & 1 \\
0.497 & 1 & 0.503 & 1 & 0.406 & 0.372 \\
0.939 & 0.655 & 0.585 & 0.646 & 0.554 & 0.492
\end{array}\right|
\end{gathered}
$$

Step5: According to the experts given weight of each index, namely $\mathrm{W}=(15,15,25,15$, $10,20)$ we can get weighted incidence matrix

$$
\mathrm{E}_{\mathrm{W}}{ }^{+}=\left|\begin{array}{cccccc}
15 & 15 & 25 & 15 & 10 & 20 \\
15 & 15 & 25 & 15 & 7.81 & 20 \\
6.615 & 10.98 & 16.85 & 9.18 & 10 & 18.42 \\
5.34 & 7.155 & 10.525 & 6.84 & 3.59 & 6.66 \\
8.01 & 6.765 & 17.475 & 6.84 & 7.32 & 14.64 \\
5.445 & 8.715 & 14.625 & 8.94 & 4.87 & 9.68 \\
15 & 15 & 25 & 15 & 10 & 20 \\
5.34 & 6.765 & 11.275 & 6.84 & 3.92 & 6.66
\end{array}\right|
$$




$\begin{array}{|cccccc|}9.36 & 7.965 & 12.925 & 9.42 & 3.59 & 6.82 \\ 15 & 13.29 & 25 & 15 & 10 & 20 \\ 7.455 & 15 & 12.575 & 15 & 4.06 & 7.44 \\ 14.085 & 9.825 & 14.625 & 9.69 & 5.54 & 9.84\end{array} \mid$

Step6: In ideal grey correlation evaluation matrix of physical and health curriculum, according to the formula (16) calculate Euclidean distance, $\left(\mathrm{D}_{1}{ }^{+}\right)^{2}=4.79,\left(\mathrm{D}_{2}{ }^{+}\right)^{2}=189.26$, $\left(\mathrm{D}_{3}{ }^{+}\right)^{2}=495.15,\left(\mathrm{D}_{4}{ }^{+}\right)^{2}=275.79,\left(\mathrm{D}_{5}^{+}\right)^{2}=407.98,\left(\mathrm{D}_{1}^{-}\right)^{2}=631.01,\left(\mathrm{D}_{2}^{-}\right)^{2}=473.04,\left(\mathrm{D}_{3}{ }^{-}\right)^{2}=2.92$, $\left(\mathrm{D}_{4}^{-}\right)^{2}=404.34485,\left(\mathrm{D}_{5}^{-}\right)^{2}=286.57$.

Step7: According to the formula (17), we can get degree of grey correlation distance

$$
\begin{aligned}
& K_{1}=\frac{\left(D_{1}^{+}\right)^{2}}{\left(D_{1}^{-}+\left(D_{1}^{+}\right)^{2}\right.}=0.00786, K_{2}=0.285498 \\
K_{3}= & 0.993975, K_{4}=0.405882, K_{5}=0.587050
\end{aligned}
$$

Step8: According to the value of grey correlation distance degree of different curriculums, we arrayed evaluation results of physical and health curriculum of 5 junior middle schools according to degree from small to large, the smaller the degree and the better results: $A_{1}, A_{2}, A_{3}, A_{4}, A_{5}$. Depending on the defined evaluation type, $\mathrm{A}_{1}$ belongs to type I; $\mathrm{A}_{2}, \mathrm{~A}_{4}$, belong to the type II; $\mathrm{A}_{5}$ belongs to the type III, $\mathrm{A}_{3}$ belongs to type IV. Idealized grey correlation distance degree of physical and health curriculum evaluation results are consistent with evaluation of both students and experts, and actual results.

\section{Conclusions}

Physical and health curriculum evaluation is foundation and content of physical education evaluation; it is very important to improve teaching level of physical education. The model of ideal grey relational distance curriculum evaluation increased accuracy and practicability, it is of great significance to improve physical and health curriculum evaluation system.

The results show that physical and health curriculum of 5 junior middle schools in Shijiazhuang city from good to bad act as: NO. 2 middle school, NO. 9 middle school, NO. 28 middle school, Hebei Normal University affiliated middle school, NO. 15 middle school. Results are consistent with evaluation of students, evaluation of experts, and actual results and make further distinguish the difference between the same types.

The model of ideal grey relational distance curriculum evaluation has significant advantages compared with the traditional methods; it provides beneficial reference for scientific physical and health curriculum evaluation. In the aspect of index value quantification, index weight definition is still need to use fractional order, AHP and the other methods going into details.

\section{References}

[1] C. X. Hong and J. Yao, "Colleges and Universities Sports Curriculum Evaluation System and Valuation Standard Research", Journal of Chengdu Sports Institute, vol. 4, no. 33, (2007), pp. 110-113.

[2] J. Liu, W. X. Zan and T. L. Jun, "A New Round of Basic Education in Our Country Sports Curriculum Ten Years Reform Review", Journal of Shanghai Sports Institute, vol. 3, no. 2, (2011), pp. 77-81.

[3] Y. F. Sheng, C. Hong and Y. X. Dong, "Sports Curriculum Quality Quantitative Evaluation Scheme Research", Journal of Chinese Sports Science and Technology, vol. 8, no. 36, (2000), pp. 13-20.

[4] I. W. Alexander, H. Sebastian and W. Arnim, "Measuring Societal Effects of Trans-disciplinary Research Projects: Design and Application of an Evaluation Method", Evaluation and Program Planning, vol. 30, no. 4, (2007), pp. 325-338.

[5] L. Tanner and W. Baron, "Integrating Participatory Elements into an Effectiveness Evaluation", Studies in Educational Evaluation, vol. 34, no. 4, (2008), pp. 201-207.

[6] M. Y. Maryati, P. Anastasia and J. P. Ray, "Investigating Evaluation Frameworks for Health Information Systems", International Journal of Medical Information, vol. 77, no. 6, (2008), pp. 377-385.

[7] A. C. Christina and C. A. Marvin, "Evaluation Theory Tree Re-examined", Studies in Educational Evaluation, vol. 34, no. 3, (2012), pp. 131-135. 
[8] J. Cui, B. Zeng and Grey Sys, "Study on Parameters Characteristics of NGM (I, I, K) Prediction Model with Multiplication Transformation", Grey Systems: Theory and Application, vol. 2, no. 3, (2012), pp. 45-50.

[9] L. Dymova, P. Sevastjanov and A. Tikhonenko, "An Approach to Generalization of Fuzzy TOPSIS Method", Information Science, no. 238, (2013), pp. 149-162.

[10] L. W. Wang, Y. Zhang and J. F. Feng, "The Euclidean Distance of Images", IEEE Transactions on Pattern Analysis and Machine Intelligence, vol. 27, no. 8, (2012), pp. 1334-1339.

[11] D. Julong, "The Grey System Theory", Huazhong University of Science and Technology Press, Wuhan, (1990). 